\title{
THE OPEN CLUSTER CHEMICAL ANALYSIS AND MAPPING SURVEY: LOCAL GALACTIC METALLICITY GRADIENT WITH APOGEE USING SDSS DR10
}

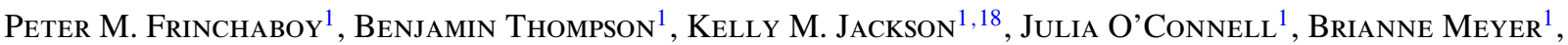 \\ Gail Zasowski $^{2,3,19}$, Steven R. Majewsir ${ }^{4}$, S. Drew Chojnowksi ${ }^{4}$, Jennifer A. Johnson ${ }^{2,3}$, Carlos Allende Prieto $^{5,6}$, \\ Timothy C. Beers ${ }^{7}$, Dmitry Bizyaev ${ }^{8}$, Howard Brewington ${ }^{8}$, Katia Cunha $^{9}$, Garrett Ebelke ${ }^{8}$, Ana Elia García Pérez ${ }^{4}$, \\ Frederick R. Hearty ${ }^{4}$, Jon Holtzman ${ }^{10}$, Karen Kinemuchi $^{8}$, Elena Malanushenko ${ }^{8}$, Viktor Malanushenko ${ }^{8}$, \\ Moses Marchante ${ }^{8}$, Szabolcs Mészáros $^{5,6}$, Demitri Muna ${ }^{2}$, David L. Nidever ${ }^{11}$, Daniel Oravetz ${ }^{8}$, Kaike Pan $^{8}$, \\ Ricardo P. Schiavon ${ }^{12}$, Donald P. Schneider ${ }^{13,14}$, Matthew Shetrone ${ }^{15}$, Audrey Simmons ${ }^{8}$, Stephanie Snedden $^{8}$, \\ Verne V. SMith ${ }^{16,17}$, AND John C. WILSON ${ }^{4}$ \\ ${ }^{1}$ Department of Physics \& Astronomy, Texas Christian University, TCU Box 298840, Fort Worth, TX 76129, USA; p.frinchaboy@ @tcu.edu, b.a.thompson1@ @cu.edu, \\ Kelly.Jackson@utdallas.edu, j.oconnell@tcu.edu,b.r.meyer@tcu.edu \\ 2 Department of Astronomy, Ohio State University, Columbus, OH 43210, USA; gail.zasowski@ gmail.com, jaj@astronomy.ohio-state.edu, \\ muna@astronomy.ohio-state.edu \\ ${ }^{3}$ Center for Cosmology and Astro-Particle Physics, Ohio State University, Columbus, OH 43210, USA \\ ${ }^{4}$ Department of Astronomy, University of Virginia, P.O. Box 400325, Charlottesville, VA 22904-4325, USA; srm4n@ virginia.edu, sdc4sb@ virginia.edu, \\ aeg4x@virginia.edu, frh3z@virginia.edu, mfs4n@virginia.edu, jcw6z@virginia.edu \\ ${ }^{5}$ Instituto de Astrofísica de Canarias, E-38205 La Laguna, Tenerife, Spain; callende@iac.es, meszi@iac.es \\ ${ }^{6}$ Departamento de Astrofísica, Universidad de La Laguna, E-38206 La Laguna, Tenerife, Spain \\ ${ }^{7}$ National Optical Astronomy Observatory and JINA (Joint Institute for Nuclear Astrophysics), Tucson, AZ 85719, USA; beers@ noao.edu \\ ${ }^{8}$ Apache Point Observatory, P.O. Box 59, Sunspot, NM 88349-0059, USA; dmbiz@ apo.nmsu.edu, hjbrew@ apo.nmsu.edu, gebelke@ apo.nmsu.edu, \\ kinemuchi@apo.nmsu.edu, elenam@apo.nmsu.edu, viktorm@apo.nmsu.edu, marchante@apo.nmsu.edu, doravetz@apo.nmsu.edu, kpan@apo.nmsu.edu, \\ asimmons@apo.nmsu.edu, sneeden@apo.nmsu.edu \\ ${ }^{9}$ Observatório Nacional, São Cristóvão, Rio de Janeiro, Brazil; cunha@email.noao.edu \\ ${ }^{10}$ Department of Astronomy, New Mexico State University, P.O. Box 30001, MSC 4500, Las Cruces, NM 88003, USA; holtz@nmsu.edu \\ ${ }^{11}$ Department of Astronomy, University of Michigan, Ann Arbor, MI 48109, USA; dnidever@umich.edu \\ ${ }^{12}$ Astrophysics Research Institute, Liverpool John Moores University, Wirral, CH41 1LD, UK; rpschiavon@ gmail.com \\ ${ }^{13}$ Department of Astronomy and Astrophysics, The Pennsylvania State University, University Park, PA 16802, USA; dps7@psu.edu \\ ${ }^{14}$ Institute for Gravitation and the Cosmos, The Pennsylvania State University, University Park, PA 16802, USA \\ ${ }^{15}$ McDonald Observatory, The University of Texas at Austin, Austin, TX 78712, USA; shetrone@ astro.as.utexas.edu \\ ${ }^{16}$ National Optical Astronomy Observatories, Tucson, AZ 85719, USA; vsmith@email.noao.edu \\ ${ }^{17}$ Steward Observatory, University of Arizona, Tucson, AZ 85721, USA \\ Received 2013 August 9; accepted 2013 August 16; published 2013 October 9
}

\begin{abstract}
The Open Cluster Chemical Analysis and Mapping (OCCAM) survey aims to produce a comprehensive, uniform, infrared-based data set for hundreds of open clusters, and constrain key Galactic dynamical and chemical parameters from this sample. This first contribution from the OCCAM survey presents analysis of 141 members stars in 28 open clusters with high-resolution metallicities derived from a large uniform sample collected as part of the Sloan Digital Sky Survey III/Apache Point Observatory Galactic Evolution Experiment. This sample includes the first high-resolution metallicity measurements for 22 open clusters. With this largest ever uniformly observed sample of open cluster stars we investigate the Galactic disk gradients of both $[\mathrm{M} / \mathrm{H}]$ and $[\alpha / \mathrm{M}]$. We find basically no gradient in $[\alpha / \mathrm{M}]$ across $7.9 \mathrm{kpc} \leqslant R_{\mathrm{GC}} \leqslant 14.5 \mathrm{kpc}$, but $[\mathrm{M} / \mathrm{H}]$ does show a gradient for $R_{\mathrm{GC}}<10 \mathrm{kpc}$ and a significant flattening beyond $R_{\mathrm{GC}}=10 \mathrm{kpc}$. In particular, whereas fitting a single linear trend yields an [M/H] gradient of $-0.09 \pm 0.03 \mathrm{dex} \mathrm{kpc}^{-1}$ — similar to previously measure gradients inside $13 \mathrm{kpc}$ - by independently fitting inside and outside $10 \mathrm{kpc}$ separately we find a significantly steeper gradient near the Sun $\left(7.9 \leqslant R_{\mathrm{GC}} \leqslant 10\right)$ than previously found $\left(-0.20 \pm 0.08 \mathrm{dex} \mathrm{kpc}^{-1}\right)$ and a nearly flat trend beyond $10 \mathrm{kpc}\left(-0.02 \pm 0.09 \mathrm{dex} \mathrm{kpc}^{-1}\right)$.
\end{abstract}

Key words: Galaxy: abundances - Galaxy: disk - Galaxy: evolution - open clusters and associations: general

Online-only material: color figures

\section{INTRODUCTION}

A key observable used to constrain galaxy evolution models and often explored in external galaxies is the variation of chemical abundances across galaxy disks. The Milky Way provides the one galaxy where we can study these variations in utmost detail using high-resolution spectroscopy. Open clusters have long been used as a key Galactic tracer to probe chemical

\footnotetext{
${ }^{18}$ Current address: Department of Physics, University of Texas-Dallas, Dallas, TX 75080, USA.

${ }^{19}$ NSF Astronomy and Astrophysics Postdoctoral Fellow.
}

and age distributions within the Milky Way disk (e.g., Carraro \& Chiosi 1994; Janes \& Phelps 1994) because they provide the most reliable "age-datable" population tracer at low latitudes. However, as traced by open clusters, the Galactic abundance "gradient" has been fit by a single linear gradient, a two-function gradient, a polynomial, or a step function. Thus, despite extensive work in this area (e.g., Bragaglia et al. 2008; Sestito et al. 2006; Jacobson et al. 2009; Pancino et al. 2010; Friel et al. 2010; Yong et al. 2012), a clear picture remains elusive, complicated by observational limitations-e.g., inhomogeneous data sets and small statistical samples, both numbers of clusters and stars per cluster (typically only 1-2 stars each). Yong 

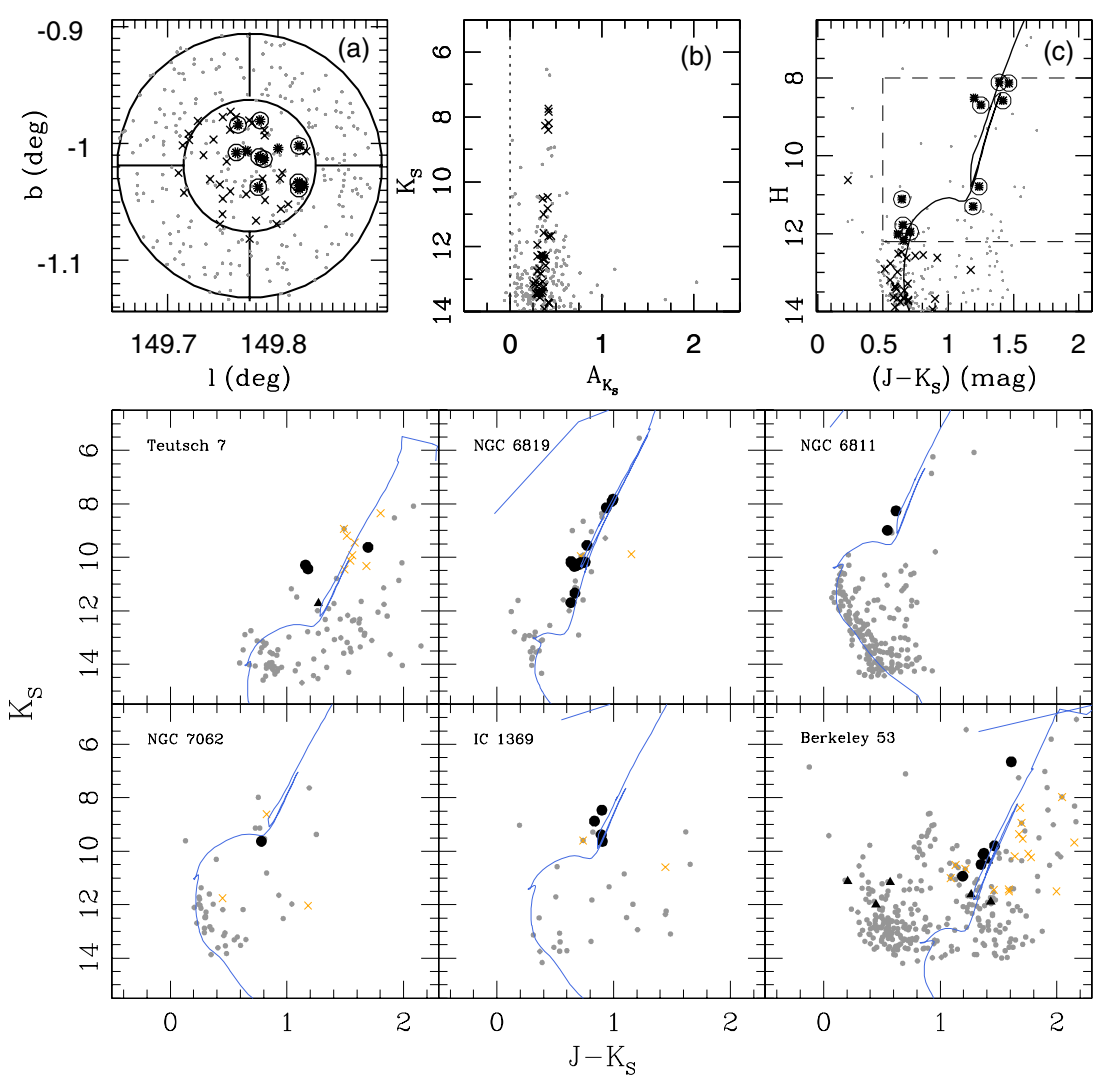

Figure 1. Example analysis for the cluster King 7 using $2 \mathrm{MASS}+W I S E$ data. (a) Galactic longitude and latitude for all stars within 2 cluster radii ( $R_{\mathrm{cl}}$; gray points); stars selected to be likely members from the extinction analysis are shown as black crosses. Stars targeted by APOGEE are circled. (b) Distribution of $A_{K_{S}}$ for all stars in the King 7 sample area; black crosses denote stars within $1.1 R_{\mathrm{cl}}$ and within the associated mean cluster $A_{K_{\mathrm{s}}}$ range. (c) Color-magnitude diagram (CMD) for all stars within $2 R_{\mathrm{cl}}$. The dashed box denotes the approximate APOGEE target selection region (however APOGEE uses the dereddened $(J-K)_{0} \geqslant 0.5$ selection), stars within the APOGEE selection are shown as black squares. The CMD is overplotted with a solar metallicity Padova Isochrone (Marigo et al. 2008) using the cluster's parameters (age, distance, reddening) from Dias et al (2002). Bottom: the 2MASS color-magnitude diagrams for all open clusters in this study. Gray points denote 2MASS stars within the cluster radius, black circles are APOGEE DR10 stars selected as members and having reliable $[\mathrm{M} / \mathrm{H}]$ and $[\alpha / \mathrm{M}]$ measurements, and black triangles are RV member stars without reliable metallicity measurements. Orange crosses are APOGEE stars that are non-members. Blue lines are Padova isochrones (Marigo et al. 2008) using the measured APOGEE [M/H] with age, distances, and reddening from Table 1.

(A color version of this figure is available in the online journal.)

et al. (2012) summarizes the state of the field: "that definitive conclusions await homogeneous analyses of larger samples of stars in larger numbers of clusters. Arguably, our understanding of the evolution of the outer disk from open clusters is currently limited by systematic abundance differences between various studies."

We aim to resolve this problem by analyzing open clusters while taking advantage of a unique, new survey, the Apache Point Observatory Galactic Evolution Experiment (APOGEE; Allende Prieto et al. 2008; Majewski et al. 2010), one of four projects included in the Sloan Digital Sky Survey III (SDSS-III; Eisenstein et al. 2011). APOGEE is an infrared (IR; $H$-band) high-resolution $(R \sim 22,500)$ survey of the Galaxy that, due to its $\sim 7 \mathrm{deg}^{2}$ field of view (Gunn et al. 2006), will eventually target stars in the fields of hundreds of open clusters in process of surveying the Galaxy. We will leverage the strength of the APOGEE cluster catalog to probe chemical trends in the Galactic disk and, in particular, the behavior of the abundances as a function of $R_{\mathrm{GC}}$ in the transition region between the solar neighborhood and the outer disk, where there remains debate about the slope and even form (e.g., linear or bilinear gradient, polynomial, or "step function") of the abundance trend (e.g., Corder \& Twarog 2001; Chen et al. 2003; Yong et al. 2005; Magrini et al. 2009; Jacobson et al. 2009; Friel et al. 2010).
In this first contribution from the Open Cluster Chemical Analysis and Mapping survey, or "OCCAM" survey we explore the local $\left(7.9 \leqslant R_{\mathrm{GC}} \leqslant 14.5\right)$ Galactic gradients of both $[\mathrm{M} / \mathrm{H}]$ and $[\alpha / \mathrm{M}]$ using data from the first of three years of the APOGEE survey.

\section{THE OPEN CLUSTER CHEMICAL ANALYSIS AND MAPPING (OCCAM) SURVEY}

The OCCAM survey goals are to create a high confidence catalog of cluster age, distance, reddening, abundances based on uniform data, and utilize this sample to make marked improvements to the detailed chemical measurement of the Milky Way disk that will inform models of galaxy evolution. The OCCAM survey will utilize large, uniform, well-calibrated surveys as its basis, starting with IR photometry from the Two Micron All-Sky Survey (2MASS; Skrutskie et al. 2006), the Spitzer/IRAC-based Galactic Legacy Infrared Mid-Plane Survey Extraordinaire programs (GLIMPSE-1, -2, -3D, 360; Benjamin et al. 2003), and the Wide-field Infrared Survey Explorer (WISE; Wright et al. 2010), combined with spectroscopy from the SDSS-III/APOGEE survey. APOGEE will provide high precision radial velocities (RVs), stellar parameters $\left(T_{\text {eff }}, \log g,[\mathrm{M} / \mathrm{H}],[\mathrm{C} / \mathrm{M}],[\mathrm{N} / \mathrm{M}],[\alpha / \mathrm{M}]\right)$, and eventually detailed abundances for individual elements $(\mathrm{Fe}, \mathrm{C}, \mathrm{N}, \mathrm{O}, \mathrm{Al}, \mathrm{Si}$, 


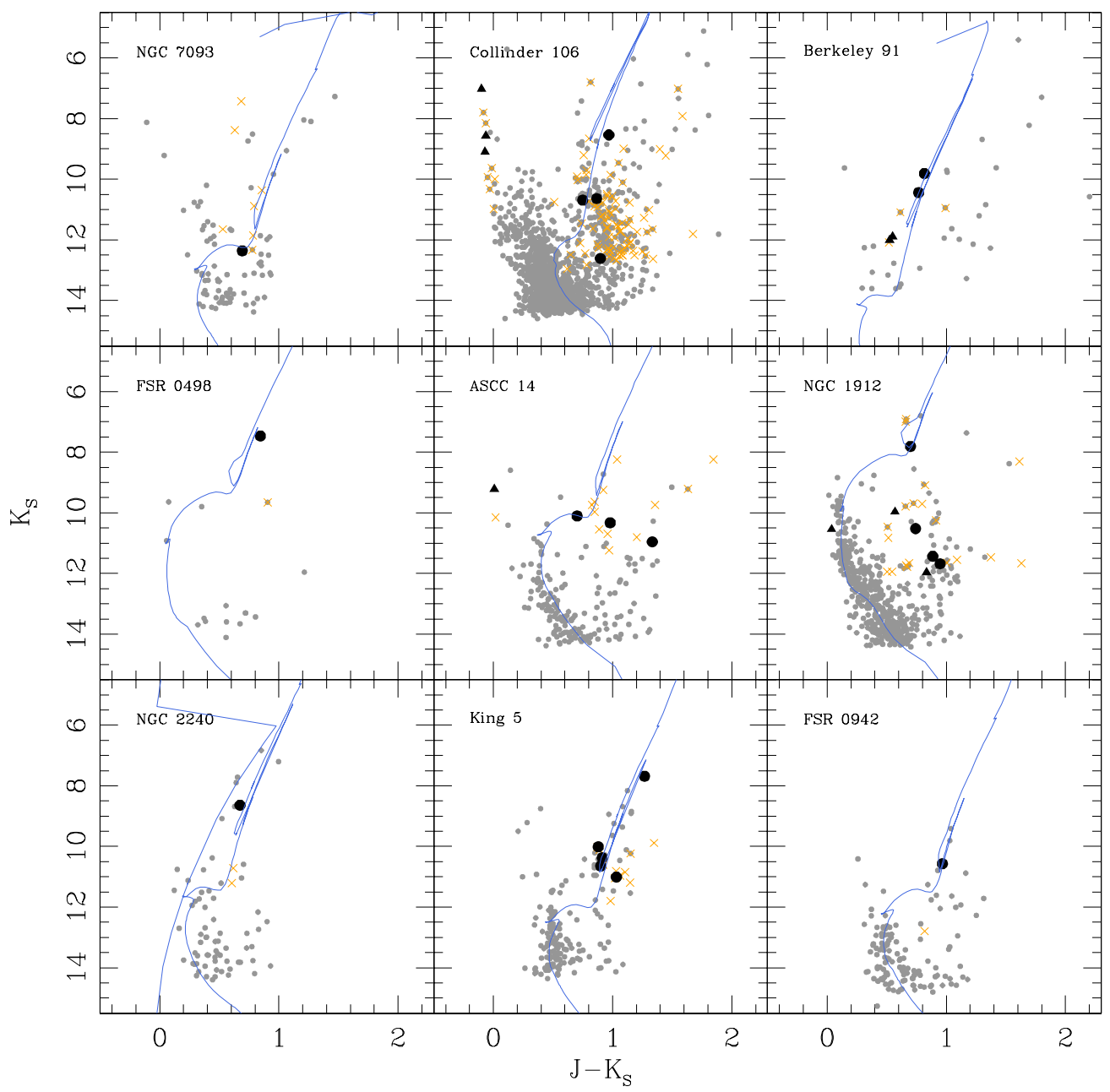

Figure 2. Same as the bottom of Figure 1.

(A color version of this figure is available in the online journal.)

$\mathrm{Ca}, \mathrm{Ni}, \mathrm{Na}, \mathrm{S}, \mathrm{Ti}, \mathrm{Mn}, \mathrm{K})$. These data sets will also be combined with new precision astrometric surveys as they come available (e.g., Pan-STARRS and Gaia; Kaiser et al. 2010; Casertano et al. 1996).

\subsection{The APOGEE Survey and SDSS Data Release 10}

The tenth data release of the SDSS (DR10; Ahn et al. 2013) provides the first public release of APOGEE data from first light observations in 2011 May through those taken in normal survey mode until 2012 July. The spectra extracted using the APOGEE data reduction pipeline, which also measures RVs (in DR10, with typical uncertainties of $150 \mathrm{~m} \mathrm{~s}^{-1}$; D. L. Nidever et al., in preparation). The stellar parameters and abundances have been determined using the APOGEE Stellar Parameters and Chemical Abundances Pipeline (ASPCAP; A. E. García Pérez et al., in preparation). ASPCAP is a set of IDL routines and a FORTRAN code called FERRE, which finds the best fit to the observed spectrum based on a $\chi^{2}$ minimization from a library of synthetic spectra computed for a large range of stellar parameters and abundances. The DR10 APOGEE database contains the stellar parameters $T_{\text {eff }}$ and $\log g$ as well as $[\mathrm{M} / \mathrm{H}]$ and $[\alpha / \mathrm{M}]$ from the ASPCAP matching and interpolation. The verification of ASPCAP was conducted by comparing its results to those of optical high-resolution studies for stars in a set of "calibration" open and globular clusters (Mészáros et al. 2013). The ASPCAP
$[\mathrm{M} / \mathrm{H}]$ provided by FERRE is well-correlated with $[\mathrm{Fe} / \mathrm{H}]$, as shown in Mészáros et al. (2013).

\section{THE OCCAM DR10 SAMPLE}

\subsection{Calibration Open Clusters}

Our study includes 6 of the 10 targeted calibration open clusters (M67, NGC 2158, NGC 2420, NGC 6791, NGC 6819, NGC 7789) from Mészáros et al. (2013). We reanalyze their stellar membership and use for them DR10 parameters consistent with those used for the other clusters in our study (Section 3.2). The calibration stars targeted by APOGEE in these clusters are flagged in the DR10 database by apogee_target $2=$ 10 (Zasowski et al. 2013).

\section{2. "Field" Open Clusters}

Because of the large SDSS field of view and the plan for APOGEE to observe all Galactic populations, but with a particular focus on the Galactic disk and bulge, the survey is targeting a large number of open clusters with a relatively small subset of the 300 fibers available per plate. To utilize these fibers efficiently, a new technique was developed to improve the chances of targeting cluster stars in these crowded, highly contaminated, low Galactic latitude fields. 


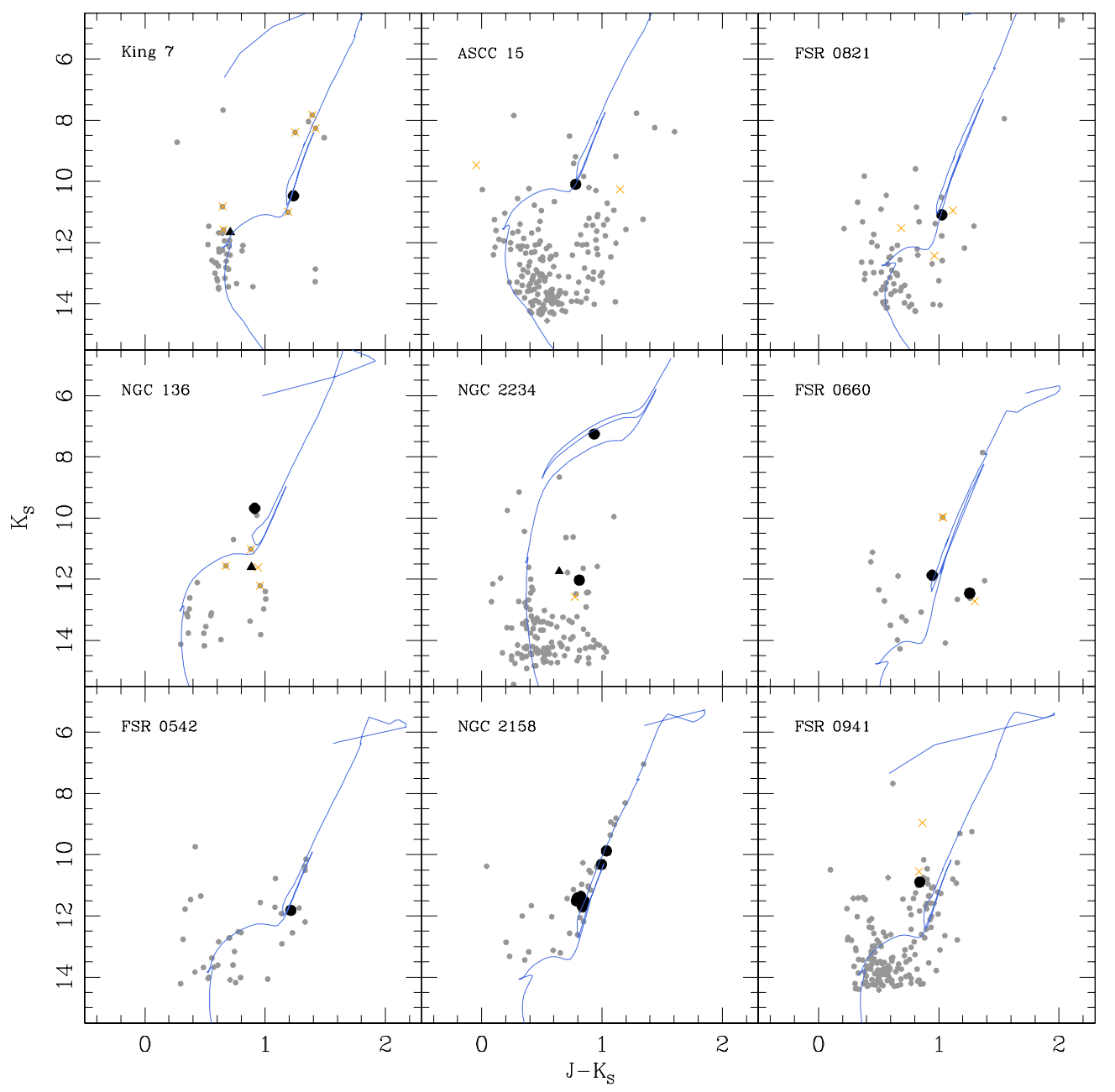

Figure 3. Same as the bottom of Figure 1.

(A color version of this figure is available in the online journal.)

This technique to isolate cluster stars from the general field uses spatial information (color-magnitude comparisons within and outside of the cataloged cluster visual radius, $R_{\mathrm{cl}}$ ) combined with filtering by reddening as derived from the Rayleigh-Jeans Color Excess technique (Majewski et al. 2011), which derives star-by-star extinctions $\left(A_{K_{S}}\right)$ that can be used to remove background and foreground stars. This method takes advantage of the fact that all stars have IR 2MASS and Spitzer/IRAC and/or WISE photometry. This IR photometry allows a direct assessment of the line-of-sight reddening to any particular star across wavelengths where the reddening law is nearly universal. At these wavelengths the color effects of reddening and stellar atmospheres are almost completely separable: the long wavelength spectral energy distributions of stars have the same Rayleigh-Jeans shape, equivalent to saying that the Vega-based, intrinsic colors of all stars are nearly constant for the correct combination of filters. Thus, the observed mid-IR colors contain information on the reddening to a star explicitly, whereas the near IR colors contain information on the stellar types. The technique identifies the cluster $A_{K}$ by maximizing the number of stars within the cluster radius relative to the number "outside" $\left(1<R_{\mathrm{cl}} \leqslant 2\right)$ of the cluster radius for a given range of $A_{K}\left(\Delta A_{K}=0.1\right)$. Given the difference of area and the sometimes non-uniform background, we measure the "outside" sample in four areas (shown in Figure 1(a)) and then have to normalize the count by the area of sky covered (e.g., square degrees)

Stars that are isolated as above and that lie within the APOGEE standard color-magnitude cuts $\left(\left(J-K_{s}\right)_{0} \geqslant 0.5\right.$ and $7 \leqslant H \leqslant 12.2$ for a standard $3 \mathrm{hr}$ APOGEE field) can then be selected as likely cluster members. For longer length APOGEE fields, fainter stars can also be targeted (down to $H=13.8$ for a $24 \mathrm{hr}$ field). This cleaning is required in most clusters for two reasons: (1) most open clusters are at low Galactic latitude and hence are heavily contaminated with field stars. (2) Due to the plate scale of the SDSS telescope and the size of the fiber ferrules, the minimum fiber-to-fiber distance is fairly large ( $\geqslant 1 \operatorname{arcmin})$, which allows for the targeting of only a handful of stars $(\sim 5-10)$ per cluster for the most distant, reddened clusters, which tend also to be the most poorly studied. This method is more fully described in P. M. Frinchaboy et al. (in preparation) and Zasowski et al. (2013). Stars targeted by APOGEE using this method are flagged in the DR 10 database by apogee_target $1=9$ (Zasowski et al. 2013).

We present a demonstration of this technique utilizing the cluster King 7, shown in Figure 1. Figure 1(a) shows the Galactic latitude and longitude area explored by our analysis. As described above, we selected likely cluster members utilizing $A_{K_{s}}$, as shown in Figure 1(b). For King 7, we find a moderate extinction to the cluster. The color-magnitude diagram (CMD) 

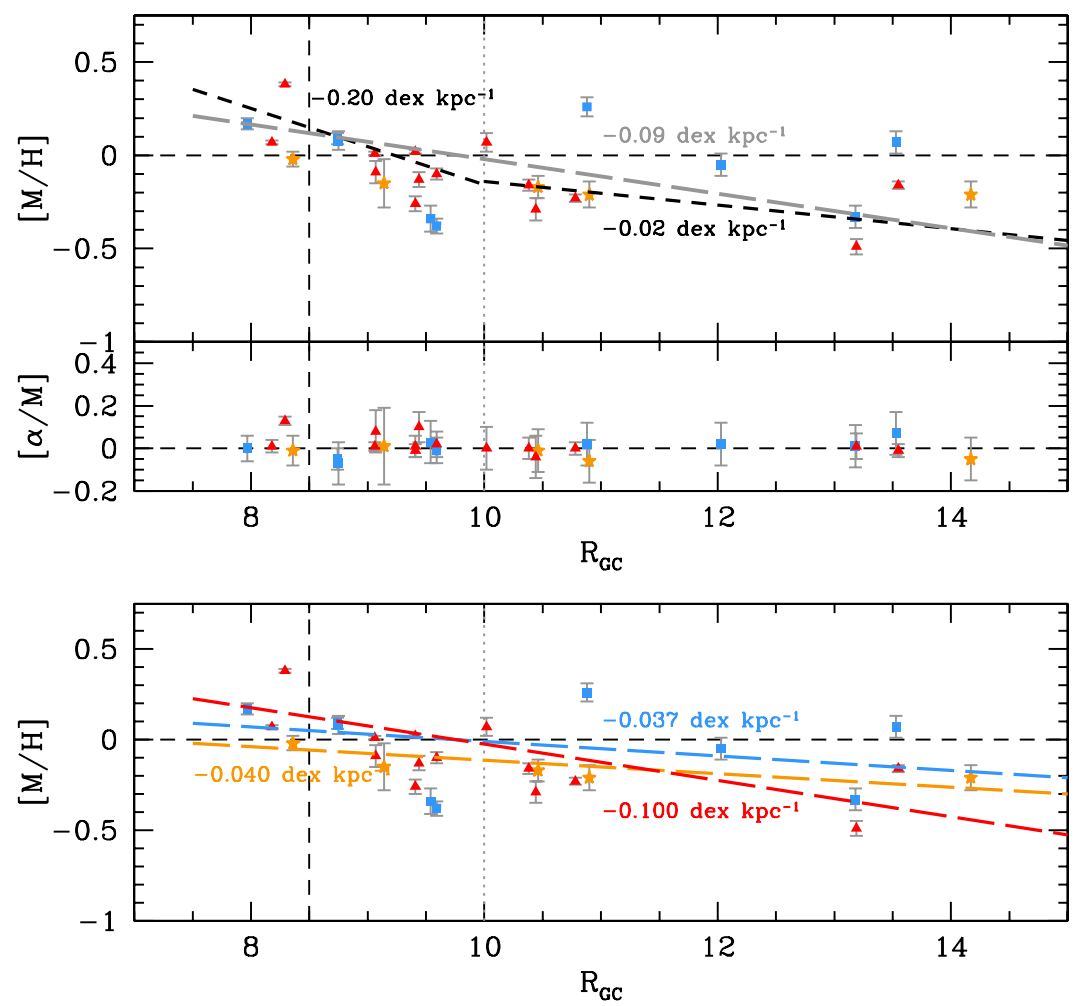

Figure 4. Top: the $[\mathrm{M} / \mathrm{H}]$ disk abundance gradient using APOGEE DR10 measurements of open clusters, assuming $R_{\odot}=8.5 \mathrm{kpc}$. A linear fit to the full sample (gray dashed line) yields a gradient of $-0.09 \pm 0.03 \mathrm{dex} \mathrm{kpc}{ }^{-1}$, which is consistent with the results of Yong et al. (2012). An alternate representation (black dashed line) is a bilinear fit broken at $R_{\mathrm{GC}}=10 \mathrm{kpc}$ : we find a gradient of $-0.20 \pm 0.08 \mathrm{dex} \mathrm{kpc}^{-1}$ for the inner cluster sample $\left(7.9 \leqslant R_{\mathrm{GC}}<10 \mathrm{kpc}\right)$ and an almost flat trend $\left(-0.02 \pm 0.09 \mathrm{dex} \mathrm{kpc}^{-1}\right)$ for the outer clusters $\left(R_{\mathrm{GC}}>10 \mathrm{kpc}\right)$. Clusters are color-coded by age: blue squares for log $($ age $)<8.5$, orange stars for $8.5 \leqslant \log ($ age $)<9.0$, and red triangles for $\log ($ age $) \geqslant 9.0$. Middle: the $[\alpha / \mathrm{M}]$ disk abundance gradient using APOGEE DR 10 open clusters. There is no significant trend in $[\alpha / \mathrm{M}]\left(-0.01 \pm 0.05 \mathrm{dex} \mathrm{kpc}^{-1}\right)$ for open clusters spanning our current sample range of $7.9 \leqslant R_{\mathrm{GC}}<14 \mathrm{kpc}$. Bottom: age gradient fits.

(A color version of this figure is available in the online journal.)

of the cluster shown in Figure 1(c) highlights the member stars having $A_{K_{s}}$ values within the selected window of extinction, and the dashed box denotes the area of APOGEE's primary target selection $\left(7.0<H<12.2\right.$ and $\left.J-K_{S} \geqslant 0.5\right)$. Finally, we compare our "cleaned" cluster CMD to the Padova isochrone matching catalog cluster values (Dias et al. 2002) for King 7 and find good agreement. By comparing the CMD with isochrone values, when available, we are able to isolate candidate open cluster stars with a high probability for membership.

\subsection{Cluster Membership and Metallicities}

To determine cluster membership, we have combined the APOGEE sample with the UCAC-4 (Zacharias et al. 2013) data set and have used the three-dimensional kinematical membership analysis from Frinchaboy \& Majewski (2008) to derive membership probabilities for each star. For this analysis we only use the RV criterion, considering cluster members as those stars with RV membership probabilities $>50 \%$, as many cluster lack stars with UCAC-4 proper motions. For clusters with a single star in the cluster radius, if it was along the Dias et al. (2002, version 3.3) catalog based isochrone fit we assumed it was a member for this study. After determining kinematical membership, a $3 \sigma$ iterative cut was made on the stellar metallicities to further remove any potential non-members.

For this paper, we analyze only reliable open clusters, which comprise clusters having member stars that clearly lie near the cluster's CMD locus (see Figures 1-3), have survey quality data in DR10, and have no ASPCAP warning flags for the analyzed stars (e.g., $4200<T_{\text {eff }}<5500 \mathrm{~K}$ and for $\log g<3.8$ ).
The clusters presented here have a median of three cluster $\mathrm{RV}$ members and one member star with reliable $[\mathrm{M} / \mathrm{H}]$ and $[\alpha / \mathrm{M}]$. A fuller description and detailed analysis for all DR10 APOGEE open clusters will be presented in P. M. Frinchaboy et al. (in preparation). Using the RV kinematic membership criteria we analyzed 546 stars, finding 141 member stars, in 28 open clusters whose bulk cluster parameters and metallicities are presented in Table 1. For 22 of these clusters, Table 1 presents the first measured high-resolution metallicity. This study increases the number of open clusters with published high-resolution metallicities by about $30 \%$.

After our membership census and after adopting the $[\mathrm{M} / \mathrm{H}]$ derived from our analysis of the APOGEE data, we refit the CMD by eye with Padova isochrones (Marigo et al. 2008) to measure the other fundamental cluster parameters (age, distance, and reddening; Figure 2, blue lines).

For most clusters, the Dias et al. (2002; version 3.3) catalog parameters were well fit, with only occasional minor corrections to the reddening required. For those clusters with a poor fit, we updated the parameters based on our refitting. Stars that clearly fall off the isochrone fits (e.g., ASCC 14, NGC 1912, NGC 2234, FSR 660) were excluded from determination of the cluster metallicity. The final cluster parameters used in the latter analysis are presented in Table 1, with updated parameters shown in italics.

\section{THE GALACTIC DISK ABUNDANCE GRADIENT}

Using the large, uniform APOGEE sample, we explore Galactic abundance gradients near and outside the solar circle 
Table 1

Selected APOGEE DR10 Open Clusters

\begin{tabular}{|c|c|c|c|c|c|c|c|c|c|c|}
\hline Cluster & $\begin{array}{c}\text { Diam. } \\
\left(^{\prime}\right)\end{array}$ & $\begin{array}{c}\log (\text { Age })^{\mathrm{a}} \\
(\mathrm{yr})\end{array}$ & $\begin{array}{l}\text { Dist. }^{\mathrm{a}} \\
(\mathrm{kpc})\end{array}$ & $E(B-V)^{\mathrm{a}}$ & $\begin{array}{c}R_{\mathrm{GC}} \\
(\mathrm{kpc})\end{array}$ & $\begin{array}{c}Z_{\mathrm{GC}} \\
(\mathrm{kpc})\end{array}$ & $\begin{array}{l}\text { Num. } \\
\text { Memb. }\end{array}$ & $\begin{array}{c}{[\mathrm{M} / \mathrm{H}]} \\
(\mathrm{dex})\end{array}$ & $\begin{array}{c}{[\alpha / \mathrm{M}]} \\
(\mathrm{dex})\end{array}$ & $\overline{\text { New? }}$ \\
\hline Teutsch 7 & 8.0 & 8.48 & 7.070 & 1.33 & 7.97 & -0.07 & 3 & $0.17 \pm 0.03$ & $-0.00 \pm 0.06$ & $\mathrm{Y}$ \\
\hline NGC 6819 & 5.0 & 9.41 & 2.432 & 0.14 & 8.18 & +0.36 & 13 & $0.07 \pm 0.01$ & $0.01 \pm 0.03$ & $\mathrm{~N}$ \\
\hline NGC 6811 & 14.0 & 8.80 & 1.215 & 0.16 & 8.36 & +0.25 & 2 & $-0.02 \pm 0.04$ & $-0.01 \pm 0.07$ & $\mathrm{Y}$ \\
\hline NGC 6791 & 10.0 & 9.92 & 5.035 & 0.14 & 8.29 & +0.95 & 29 & $0.38 \pm 0.01$ & $0.13 \pm 0.02$ & $\mathrm{~N}$ \\
\hline NGC 7062 & 5.0 & 8.46 & 2.100 & 0.51 & 8.75 & -0.10 & 1 & $0.08 \pm 0.05$ & $-0.07 \pm 0.10$ & $\mathrm{Y}$ \\
\hline Berkeley 53 & 22.0 & 9.09 & 3.100 & 1.40 & 9.07 & +0.20 & 6 & $-0.09 \pm 0.06$ & $0.08 \pm 0.10$ & $\mathrm{Y}$ \\
\hline NGC 7093 & 9.0 & 8.95 & 3.200 & 0.50 & 9.14 & -0.24 & 1 & $-0.15 \pm 0.13$ & $0.01 \pm 0.18$ & $\mathrm{Y}$ \\
\hline NGC 2682 & 25.0 & 9.45 & 0.792 & 0.04 & 9.06 & +0.42 & 21 & $0.01 \pm 0.01$ & $0.01 \pm 0.02$ & $\mathrm{~N}$ \\
\hline NGC 7789 & 25.0 & 9.15 & 1.795 & 0.28 & 9.41 & -0.17 & 16 & $0.02 \pm 0.01$ & $-0.01 \pm 0.03$ & $\mathrm{~N}$ \\
\hline Collinder 106 & 35.0 & 9.90 & 1.000 & 0.40 & 9.41 & -0.01 & 3 & $-0.26 \pm 0.04$ & $0.01 \pm 0.05$ & $\mathrm{Y}$ \\
\hline Berkeley 91 & 3.0 & 9.35 & 4.100 & 0.12 & 9.44 & +0.02 & 2 & $-0.13 \pm 0.04$ & $0.10 \pm 0.07$ & $\mathrm{Y}$ \\
\hline FSR 498 & 1.5 & 8.55 & 1.800 & 0.22 & 9.54 & -0.01 & 1 & $-0.34 \pm 0.07$ & $0.03 \pm 0.10$ & $\mathrm{Y}$ \\
\hline NGC 1912 & 20.0 & 8.50 & 1.100 & 0.35 & 9.59 & +0.01 & 1 & $-0.38 \pm 0.04$ & $-0.01 \pm 0.06$ & $\mathrm{Y}$ \\
\hline NGC 2240 & 11.0 & 9.20 & 1.551 & 0.04 & 10.02 & +0.32 & 1 & $0.07 \pm 0.05$ & $0.00 \pm 0.10$ & $\mathrm{Y}$ \\
\hline King 5 & 14.2 & 9.10 & 2.230 & 0.67 & 10.38 & -0.17 & 4 & $-0.16 \pm 0.03$ & $0.00 \pm 0.05$ & $\mathrm{Y}$ \\
\hline FSR 942 & 8.0 & 9.00 & 2.000 & 0.80 & 10.44 & -0.13 & 1 & $-0.29 \pm 0.06$ & $-0.04 \pm 0.10$ & $\mathrm{Y}$ \\
\hline King 7 & 7.0 & 8.82 & 2.200 & 1.25 & 10.46 & -0.04 & 1 & $-0.17 \pm 0.06$ & $-0.01 \pm 0.10$ & $\mathrm{Y}$ \\
\hline NGC 2420 & 5.0 & 9.30 & 2.480 & 0.06 & 10.78 & +0.83 & 11 & $-0.23 \pm 0.02$ & $0.00 \pm 0.03$ & $\mathrm{~N}$ \\
\hline ASCC 15 & 24.0 & 8.60 & 2.400 & 0.40 & 10.88 & +0.00 & 1 & $0.26 \pm 0.05$ & $0.02 \pm 0.10$ & $\mathrm{Y}$ \\
\hline FSR 821 & 9.9 & 8.80 & 2.400 & 0.85 & 10.90 & -0.01 & 1 & $-0.21 \pm 0.07$ & $-0.06 \pm 0.10$ & $\mathrm{Y}$ \\
\hline NGC 136 & 4.0 & 8.40 & 5.220 & 0.70 & 12.03 & -0.12 & 1 & $-0.05 \pm 0.06$ & $0.02 \pm 0.10$ & $\mathrm{Y}$ \\
\hline NGC 2234 & 8.0 & 7.70 & 4.800 & 1.00 & 13.18 & +0.24 & 1 & $-0.33 \pm 0.06$ & $0.01 \pm 0.10$ & $\mathrm{Y}$ \\
\hline FSR 660 & 2.6 & 9.22 & 5.126 & 0.79 & 13.19 & -0.05 & 1 & $-0.49 \pm 0.04$ & $0.01 \pm 0.06$ & $\mathrm{Y}$ \\
\hline FSR 542 & 8.0 & 8.50 & 6.600 & 1.10 & 13.53 & +0.04 & 1 & $0.07 \pm 0.06$ & $0.07 \pm 0.10$ & $\mathrm{Y}$ \\
\hline NGC 2158 & 5.0 & 9.02 & 5.071 & 0.50 & 13.55 & +0.16 & 11 & $-0.16 \pm 0.02$ & $-0.01 \pm 0.03$ & $\mathrm{~N}$ \\
\hline FSR 941 & 12.0 & 8.70 & 5.800 & 0.70 & 14.17 & -0.08 & 1 & $-0.21 \pm 0.07$ & $-0.05 \pm 0.10$ & $\mathrm{Y}$ \\
\hline
\end{tabular}

Note. ${ }^{a}$ Dias et al. (2002) catalog (version 3.3 - jan/10/2013). Italics denote refitted values and that differ from Dias et al.

( $7.9 \leqslant R_{\mathrm{GC}} \leqslant 14.5$; we assume $R_{\text {Sun }}=8.5 \mathrm{kpc}$ ). All clusters in the sample have $|Z|<500 \mathrm{pc}$, except NGC $2420(Z=830 \mathrm{pc})$ and NGC $6791(Z=950 \mathrm{pc})$. In this contribution, we analyze the Galactic trends in metallicity $([\mathrm{M} / \mathrm{H}])$ and $\alpha$-abundances $([\alpha / \mathrm{M}])$ only.

\section{1. $[M / H]$ Trends}

Using distances and metallicities from Table 1, we assess Galactic disk metallicity trends, as shown in Figure 4. Fitting a linear trend to our full sample, we find a gradient of $-0.09 \pm$ $0.03 \mathrm{dex} \mathrm{kpc}^{-1}$ (see Figure 4, top), which is steeper than, but consistent within the errors of previous gradients derived from literature compilations of open clusters, e.g., $-0.06 \pm$ $0.02 \mathrm{dex} \mathrm{kpc}^{-1}$ found by Pancino et al. (2010) and Friel et al. $(2002,2010)$, and is closer to the gradient found by Yong et al. $\left(2012 ;-0.09 \pm 0.01 \mathrm{dex} \mathrm{kpc}^{-1}\right)$.

We also investigated an alternate fit by independently fitting inside and outside $R_{\mathrm{GC}}=10 \mathrm{kpc}$, which is near the dynamical signature for Galactic co-rotation (Lépine et al. 2013). This yields a significantly steeper gradient $\left(-0.20 \pm 0.08 \mathrm{dex} \mathrm{kpc}{ }^{-1}\right)$ near the Sun $\left(7.9 \leqslant R_{\mathrm{GC}} \leqslant 10 \mathrm{kpc}\right)$ than previously reported (Figure 3(a)). This inner gradient is steeper than those previously found, even for "broken"/multi-linear fits-e.g., Yong et al. (2012) measured a gradient of $-0.09 \pm 0.01 \mathrm{dex} \mathrm{kpc}^{-1}$ for $R_{\mathrm{GC}}<13 \mathrm{kpc}$, a similar trend to that from our full sample fit, which covers a similar $R_{\mathrm{GC}}$ range.

We find little gradient in our sample beyond $R_{\mathrm{GC}}>10 \mathrm{kpc}$ $\left(-0.02 \pm 0.09 \mathrm{dex} \mathrm{kpc}^{-1}\right)$, where a plateau of $\langle[\mathrm{M} / \mathrm{H}]\rangle=-0.3$ appears. This plateau is similar to the flattening found by Yong et al. (2012) for clusters beyond $R_{\mathrm{GC}}=12 \mathrm{kpc}$.

\section{2. $[\alpha / M]$ Trends}

We find little to no trend in $[\alpha / \mathrm{M}]\left(-0.01 \pm 0.05 \mathrm{dex} \mathrm{kpc}^{-1}\right)$ from analysis of the APOGEE DR10 open cluster sample across the full range of Galactic radius $\left(7.9 \leqslant R_{\mathrm{GC}} \leqslant 14.5 \mathrm{kpc}\right.$; Figure 4, middle). While the sample does not yet include the most distant clusters (e.g., Berkeley 29 and Saurer 1) that have been shown to be $\alpha$-enhanced, we should be able to place further constraints on this trend with data from future APOGEE data releases, which target more distant clusters.

\subsection{Time Evolution of the Gradient?}

The large age span of our sample allows us to explore dependencies of the gradients with age. We divided the sample into three age bins: nine "young" clusters $(\log ($ age $)<8.5)$, five "intermediate" clusters $(8.5 \leqslant \log ($ age $)<9.0)$, and fourteen "old" clusters (log(age) $\geqslant 9.0$; see Figure 4 , bottom). We fit the gradient as a function of $R_{\mathrm{GC}}$ using only clusters from each age bin and find similar behavior for the "young" $(\Delta[\mathrm{M} / \mathrm{H}]=$ $\left.-0.04 \pm 0.09 \mathrm{dex} \mathrm{kpc}^{-1}\right)$ and "intermediate" $(\Delta[\mathrm{M} / \mathrm{H}]=$ $-0.04 \pm 0.15 \mathrm{dex} \mathrm{kpc}^{-1}$ ) samples. The "young" sample is small and shows a large scatter, which could be intrinsic, or given that APOGEE is tuned to giant stars these could be due to less reliable parameters (e.g., the star in FSR 542 is near the $\log g$ warning limit in DR10) or that the one star selected could actually be a non-member. The "old" sample shows a slightly steeper gradient $\left(\Delta[\mathrm{M} / \mathrm{H}]=-0.10 \pm 0.04 \mathrm{dex} \mathrm{kpc}^{-1}\right)$, which hints that there 
may be some evolution as a function of age - a shallowing of the gradient with time. We caution against significant interpretation given the limited $R_{\mathrm{GC}}$ sampling of the present study. With a larger sample from future APOGEE data releases, we should be able to place tighter constraints on the age trends in the Galactic disk.

\section{CONCLUSIONS}

We describe the first results from the OCCAM collaboration's exploration of the SDSS-III/APOGEE open cluster data as presented in DR10.

1. The SDSS-III/APOGEE DR10 data set contains the largest sample of uniformly observed and analyzed high-resolution metallicity measurement of stars in open clusters, (141 member stars in 28 clusters), including the first highresolution metallicity measurements for 22 open clusters.

2. For this sample of clusters, spanning $7.9<R_{\mathrm{GC}}<$ $10 \mathrm{kpc}$ we find a steep inner $[\mathrm{M} / \mathrm{H}]$ gradient, $-0.20 \pm$ $0.08 \mathrm{dex} \mathrm{kpc}^{-1}$, but a basically flat trend $(-0.02 \pm$ $0.09 \mathrm{dex} \mathrm{kpc}{ }^{-1}$ ) beyond $R_{\mathrm{GC}}=10 \mathrm{kpc}$. This inner gradient is steeper than found by previous measurements (Friel et al. 2010; Yong et al. 2012). The location of the split in these two samples is near the Galactic co-rotation radius, similar to the break in abundance gradient found by Lépine et al. (2013) using Cepheids. This feature may provide a useful constraint for studies of galaxy evolution.

3. We find no significant $[\alpha / \mathrm{M}]$ trend over $7.9<R_{\mathrm{GC}}<$ $14.5 \mathrm{kpc}$. However, other studies that have found a trend toward the outer disk (e.g., Yong et al. 2012) have included more distant clusters than presented here (e.g., Berkeley 29 and Saurer 1 at $R_{\mathrm{GC}} \sim 20 \mathrm{kpc}$ ).

Future OCCAM publications will present the full, detailed analysis of the entire DR10 open cluster sample, including a reanalysis of the clusters' fundamental parameters (age, distance, and reddening) and additional chemical elements anticipated by the full APOGEE data release DR12 (2014 December).

P.M.F. acknowledges funding from the TCU RCAF and JFSRP programs. K.J. acknowledges funding from a TCU SERC grant. K.C. acknowledges support for this research from the National Science Foundation (AST-0907873). Funding for SDSS-III has been provided by the Alfred P. Sloan Foundation, the Participating Institutions, the NSF, and the U.S. Department of Energy Office of Science. The SDSS-III Web site is http://www.sdss3.org/.

SDSS-III is managed by the Astrophysical Research Consortium for the Participating Institutions of the SDSS-III
Collaboration including the University of Arizona, the Brazilian Participation Group, Brookhaven National Laboratory, University of Cambridge, Carnegie Mellon University, University of Florida, the French Participation Group, the German Participation Group, Harvard University, the Instituto de Astrofisica de Canarias, the Michigan State/Notre Dame/JINA Participation Group, Johns Hopkins University, Lawrence Berkeley National Laboratory, Max Planck Institute for Astrophysics, New Mexico State University, New York University, Ohio State University, Pennsylvania State University, University of Portsmouth, Princeton University, the Spanish Participation Group, University of Tokyo, University of Utah, Vanderbilt University, University of Virginia, University of Washington, and Yale University.

Facility: Sloan

\section{REFERENCES}

Ahn, C. P., Anderson, S. F., Anderton, T., et al. 2013, ApJ, submitted (arXiv:1307.7735)

Allende Prieto, C., Majewski, S. R., Schiavon, R., et al. 2008, AN, 329, 1018

Benjamin, R. A., Churchwell, E., Babler, B. L., et al. 2003, PASP, 115, 953

Bragaglia, A., Sestito, P., Villanova, S., et al. 2008, A\&A, 480, 79

Carraro, G., \& Chiosi, C. 1994, A\&A, 287, 761

Casertano, S., Lattanzi, M. G., Perryman, M. A. C., \& Spagna, A. 1996, Ap\&SS, 241, 89

Chen, L., Hou, J. L., \& Wang, J. J. 2003, AJ, 125, 1397

Corder, S., \& Twarog, B. A. 2001, AJ, 122, 895

Dias, W. S., Alessi, B. S., Moitinho, A., Lépine, J. R. D., \& Alessi, B. S. 2002, A\&A, 389, 8718

Eisenstein, D. J., Weinberg, D. H., Agol, E., et al. 2011, AJ, 142, 72

Friel, E. D., Jacobson, H. R., \& Pilachowski, C. A. 2010, AJ, 139, 1942

Friel, E. D., Janes, K. A., Tavarez, M., et al. 2002, AJ, 124, 2693

Frinchaboy, P. M., \& Majewski, S. R. 2008, AJ, 136, 188

Gunn, J. E., Siegmund, W. A., Mannery, E. J., et al. 2006, AJ, 131, 2332

Jacobson, H. R., Friel, E. D., \& Pilachowski, C. A. 2009, AJ, 137, 4753

Janes, K. A., \& Phelps, R. L. 1994, AJ, 108, 1773

Kaiser, N., Burgett, W., Chambers, K., et al. 2010, Proc. SPIE, 7773, 12

Lépine, J. R. D., Andrievky, S., Barros, D. A., Junqueira, T. C., \& Scarano, S. 2013, in IAU Symp. 298 (arXiv:1307.7781)

Magrini, L., Sestito, P., Randich, S., \& Galli, D. 2009, A\&A, 494, 95

Majewski, S. R., Wilson, J. C., Hearty, F., Schiavon, R. R., \& Skrutskie, M. F. 2010, in IAU Symp. 265, Chemical Abundances in the Universe: Connecting First Stars to Planets, ed. K. Cunha, M. Spite, \& B. Barbuy (Paris: IAU), 480

Majewski, S. R., Zasowski, G., \& Nidever, D. L. 2011, ApJ, 739, 25

Marigo, P., Girardi, L., Bressan, A., et al. 2008, A\&A, 482, 883

Mészáros, Sz., Holtzman, J., García Pérez, A. E., et al. 2013, AJ, in press (arXiv:1308.6617)

Pancino, E., Carrera, R., Rossetti, E., \& Gallart, C. 2010, A\&A, 511, A56

Sestito, P., Bragaglia, A., Randich, S., et al. 2006, A\&A, 458, 121

Skrutskie, M. F., Cutri, R. M., Stiening, R., et al. 2006, AJ, 131, 1163

Wright, E. L., Eisenhardt, P. R. M., Mainzer, A., et al. 2010, AJ, 140, 1868

Yong, D., Carney, B., \& de Almeida, M. L. T. 2005, AJ, 130, 597

Yong, D., Carney, B. W., \& Friel, E. D. 2012, AJ, 144, 95

Zacharias, N., Finch, C. T., Girard, T. M., et al. 2013, AJ, 145, 44

Zasowski, G., Johnson, J. A., Frinchaboy, P. M., et al. 2013, AJ, 146, 81 